\title{
Teaching Strategies for Graduates with Different Specialty Background in Biomedical Signal Processing Course
}

\author{
Nini Rao ${ }^{1, a}$, Dingyun Liu ${ }^{1, b}$ and Wei Zeng ${ }^{1,2, c}$ \\ 1Department of Biomedical Engineering, School of Life Science and Technology, University of \\ Electronic Science and Technology of China, Chengdu, China \\ 2Department of Biomedical Engineering, School of Automation and Electronic Information, \\ Sichuan University of Science and Engineering, Zigong, China \\ a'Raonn@uestc.edu.cn \\ b932980301@qq.com \\ cberrystraw@163.com
}

Keywords: Biomedical Signal Processing, course, specialty background, teaching strategy.

\begin{abstract}
Biomedical signal processing is one of key courses in the major of Biomedical Engineering. The teaching contents of this course are involved in multiple subject fields, such as biology, medicine, signal processing, computer science and even mathematics/physics. Most graduates studying this course usually have a single of subject background. Thus, it is a huge of challenge to realize the teaching objectives of this course. This paper proposed five strategies to improve the teaching effects of this course. The teaching practice showed that the proposed methods are feasible and effective.
\end{abstract}

\section{Introduction}

Biomedical Engineering is an interdisciplinary subject involving in life science, medical science and engineering technology. Since Biomedical Engineering major was established, courses and laboratories have been proposed and implemented to enhance the efficacy of communication channels between the biomedical and engineering disciplines [1-2], in which biomedical signal processing is one of key courses [3]. A lot of reaseches have been shown that signal processing is indeed a very useful technology/method in finding solusions of wide biomedical problems. For example, Myers et al. [4] attempted to use the power spectral density function as a noninvasive predictor of sudden cardiac death (SCD). Their results suggest that the power spectral component between $0.35-0.5 \mathrm{~Hz}$ provids the best discrimination bewteen patient groups compared to other power and nonpower spectral parameters. Tiwari et al. [5] predicted the probable gene using Fourier analysis of genomic sequences. They find that the relative height of the peak at $f=1 / 3$ in the Fourier spectrum of DNA sequences is a good discriminator of coding regions. EI-Gohary et al. [6] proposed a whitened cross-correlation analysis to establish causality between medical signals. This new signal processing method can identify or rule out possible causal relationships and often leads to new insights that are impossible to discern from the traditional cross-correlation analysis. Subha et al. [7] discussed different signal processing methods that are used to extract the hidden information from EEG (Electroencephalogram) signal, including linear, frequency domain, time - frequency and non-linear techniques like correlation dimension (CD), largest Lyapunov exponent (LLE), Hurst exponent (H), different entropies, fractal dimension(FD), Higher Order Spectra (HOS), phase space plots and recurrence plots.

The primary objectives of biomedical signal processing course are instill in the student a qualitative understanding of signal processing as an essential component of knowledge for a biomedical engineering and to develop the student's skills in applying these concepts quantitatively to biomedical problems [8]. Usually, most graduates who study this course have different specialty background in our university. For example, some graduates come from the major of Biomedical Engineering, who 
have the best foundation learning this course. Some come from Electrical Engineering, Computer Science, Medicine or Biology. It is difficult for students only with the background of Electronic Engineering/Computer Science to understand the biomedical problems to be solved, whereas the students with the backgrounds of biology/medicine are difficult to master the engineering technology/methods. The teaching contents of biomedical signal processing course are very wide [9-10], which are involved in biology, medicine, signal processing technology, computer technology and etc. Thus, it is a huge of challenge to make each student possess the target skills after studying this course. To this end, this paper proposed five strategies to improve the teaching effects of this course and gave the teaching practice results after carrying out the proposed strategies.

\section{The proposed teaching strategies}

The teaching links of biomedical signal processing course mainly include the lecturing, the seminar, the curriculum design after lecturing and the final examination. We proposed the following strategies to improve the teaching effects of the important links above.

Strategy 1: Teaching basic theories of signal processing using a few of teaching hours for establishing basic concepts for the students with the background of biology/medicine. For example, what are digital signal and system, the relationships among an input signal, an output signal and a system function, auto and cross-correlations, Fourier Transformation and so on. Since this part of students is very difficult to understand signal processing technology, the teacher should give great patience in teaching.

Strategy 2: Adding and extending the knowledge of biology/medicine for helping the students with engineering background to understand the biomedical problems to be solved. For example, the solved problem is to enhance fetal electrocardiogram (ECG). Combining this problem, the teacher should introduce heart's structure, the methods collecting ECG signal and the characteristic waves of ECG as well as the methods locating the P, QRS and T waves in an ECG recording.

Strategy 3: In the seminar, the students with the background of biology/medicine are invited to introduce the knowledge involved in the biomedical problems. The students with engineering background are invited to deliver the signal processing methods/algorithms. Through a seminar, each student can learn a lot of knowledge who previously lacks.

Strategy 4: In the curriculum design after lecturing, each group is arranged to be consisted of the students with biology/medicine, engineering and computer background. Through jointly analyzing and solving the problem, different backgrounds of students can learn each other and finish the homework well.

Strategy 5: Two final examination papers with the same degrees of difficulty and scores are designed to evaluate whether the teaching goal of course is reached. A student is required to select one of them to answer all the test questions in a test paper. One focuses on testing the ability applying signal processing technologies/tools to solve biological or medical problems and requires deeper biological/medical explanation for results, which is probably selected by most students with biology/medicine major. Other the ability developing new signal processing technologies/tools to solve biological or medical problems, which is probably selected by most students with engineering background. Usually how to test decides how to learn[11]. Thus, the proposed examination strategy can take an important role in the study way of a student and the selection of teaching contents.

\section{The teaching practice}

In our school, the total teaching time of Biomedical Signal Processing course is 40 hours and 4 teaching hours are arranged in a week. The teaching contents consist of eight lectures, including introduction, reviewing basic signal processing technology, correlation between biomedical signals, power spectra of biomedical signals, enhance of biomedical signals, prediction of potential variables in a biomedical system, multiple variable analysis of biomedical signals and time-frequency analysis 
of biomedical signals. Before beginning the teaching, we first used questionnaire survey to obtain the major distribution of students, as shown in Table 1 and then divided study groups according to their majors.

Table 1 the major distribution of students in 2014

\begin{tabular}{|c|c|c|c|c|c|c|}
\hline \multirow{2}{*}{ Total number } & \multicolumn{6}{|c|}{ The major distribution (\%) } \\
\cline { 2 - 7 } & BME & B/M & EE & CS & M/P & Other \\
\hline 70 & 31.43 & 18.57 & 32.86 & 8.57 & 4.29 & 4.28 \\
\hline
\end{tabular}

BME: Biomedical Engineering; B/M: Biology/Medicine; EE: Electrical Engineering; CS:

Computer Science; M/P: Mathematics/Physics.

From Table 1, we know that the students in EE, CS, M/P, etc., which take up 50\%, probably are difficult to understand biological or medical problems since they previously did not learn the related courses. The students in B/M, CS, M/P and other majors, which take up 35.71, probably are difficult to understand signal processing methods since they have not the theoretical foundation of signal processing, such as signal and system and digital signal processing. This survey guides the teaching well.

Next, the strategies above were applied to the teaching practice of this course during Feb., 2014 to May, 2014. After finishing the teaching, we finally compared the scores of students' examination and teaching estimation, which are made by the students and experts from the teaching quality committee of school and university, with those in 2012 and 2013. The results are shown in Table 2.

Table 2 The scores of examination and estimation after/before the strategies

\begin{tabular}{|c|c|c|c|}
\hline & 2012 & 2013 & 2014 \\
\hline Average score of examination & 69 & 70 & 75 \\
\hline Score of teaching Estimation & 82 & 83 & 89 \\
\hline
\end{tabular}

From Table 2, the average scores of students' examination are greatly enhanced after applying the strategies and so the learning effects are improved. The teaching was estimated as "excellence" (the score more than 85 is defined as "excellence") and the teacher received an honor certificate since of excellent teaching quality in 2014. The students' and teacher's win-win is achieved after applying the strategies.

\section{Summary}

Graduates who learn Biomedical Signal Processing course have usually different specialty background. In order to improve the teaching effect of this course, this paper proposed five strategies and applied them to the teach practice of this course. The practical results showed that the proposed methods are feasible and effective in improving the teaching of this course.

\section{Acknowledgements}

This work was financially supported by the National Natural Science Foundation of China (81171411) and National Quality Course Construction Project of China (Y02025023701022).

\section{References}

[1] M. B. Silver-Thor: A rehabilitation engineering course for biomedical engineers, IEEE Trans. Educ., vol. 45, no.4 (2002), p.299-306.

[2] P. T. Bhatti and J. H. McClellan: A cochlear implant signal processing lab: Exploration of a problem-based learning exercise, IEEE Trans. Educ., vol. 54 (2011), no. 4, p. 628-636. 
[3] J. Nagel, in: Biomedical Engineering Education in Europe-Status Reports, BIOMEDEA, Germany (2005).

[4] G. A. Myers, G. J. Martin, N. M. Magid, et al.: Power spectral analysis of heart rate variability in sudden cardiac deaths, IEEE Tans. Biomed. Eng. BME-33 (1986), p. 1149-1158.

[5] S. Tiwari, S. Ramachandran, A. Bhattacharya, et al.: Prediction of probable genes by Fourier analysis of genomic sequences, CABIOS, vol. 13, no. 3 (1997), p. 263-270.

[6] M. EI-Gohary and J. McNames: Establishing causality with whitened cross-correlation analysis, IEEE Trans. BME, vol. 54, No. 12 (2007), p. 2214-2222.

[7] D. P. Subha, P. K. Joseph, U. R. Acharya, C. M. Lim: EEG signal analysis: a survey, J Med Syst., vol. 34, no. 2 (2010), p.195-212.

[8] A. M. Attikiouzel: Biomedical Signal Processing: Present and Future, Proc. of the Fifth Int. Symposium on Signal Processing and Its Applications, Vol. I (1999), p. 2.

[9] M. Akay, in: Biomedical Signal Processing, Academic Press, (2001).

[10] S. Cerutti, C. Marchesi. Advanced Methods of Biomedical Signal Processing, The Institute of Electrical and Electronics Engineers, Inc., (2011).

[11] X. H. Xie, X.H. Chen, et al: On the Reform of Curriculum Examination under the Management of Credit System (In Chinese), University Education Science, vol. 1 (2006), p.31-32. 